\title{
In situ Phosphorus-doped Polysilicon for Excitation and Detection in Micromechanical Resonators
}

\author{
SIEBE BOUWSTRA, EDDY DE WEERD and MIKO ELWENSPOEK
}

Sensors and Actuators Research Unit, University of Twente, P.O. Box 217, 7500 AE Enschede (The Netherlands)

(Received December 19, 1989; in revised form June 7, 1990; accepted June 28, 1990)

\begin{abstract}
Low-pressure chemical vapour deposited (LPCVD) in situ phosphorus-doped polysilicon films have been grown from a $60: 30: 300 \mathrm{sccm}$ silane:phosphine (2000 ppm):nitrogen mass-flow mixture at $625^{\circ} \mathrm{C}$ under varied process conditions. Thickness uniformity, grain size, dopant concentration, resistivity, temperature coefficient of resistivity, longitudinal strain gauge factor and the temperature coefficient of the gauge factor are determined. A growth rate with a non-uniformity $(3 \sigma)$ of $5 \%$ is obtained, yielding films with a grain size of $20-30 \mathrm{~nm}$ and a surface roughness of $12 \mathrm{~nm}$ (peak-to-valley heights), boṭh before and after annealing, and a dopant concentration of $(2-3) \times 10^{20} \mathrm{~cm}^{-3}$. Resistivities of the order of $1 \mathrm{~m} \Omega \mathrm{cm}$ can be obtained with a temperature coefficient close to zero after annealing at $900^{\circ} \mathrm{C}$ for $30 \mathrm{~min}$, with a longitudinal gauge factor of -20 and a temperature coefficient of the gauge factor of $-0.25 \% /{ }^{\circ} \mathrm{C}$. A mechanism incorporating the diffusion of dislocations during annealing is proposed to explain the observed effect. The films are appropriate for application as resistors for thermal excitation and piezoresistive detection in resonating micromechanical devices.
\end{abstract}

\section{Introduction}

Polycrystalline silicon (polysilicon) has been used in integrated circuits, mainly for the gate electrodes in MOS devices, for two decades. It is also used for circuit interconnections, for ohmic contacts, for high-value resistors and for diffusion sources $[1,2]$. Polysilicon is now also used in solid-state sensors $[2,3]$ as a strain gauge material [4-9] and as a temperature-sensitive material [10]. Polysilicon deposited at low temperatures is used as a construction material in solid-state sensors $[4,10,11]$ and actuators $[12-16]$ because of its possible small tensile residual stress $[17,18]$. Recently, we have started using polysilicon for thermal excitation and piezoresistive detection in a resonating micromechanical structure $[19,20]$.
Resonating micromechanical structures consist of an element for the excitation and an element for the detection of the vibration of the structure. These elements can be an integral part of the structure, see Fig. 1, with an excitation element for exerting a bending moment, and a detection element to detect bending strain. One technique makes use of resistive heating for thermal excitation and resistive strain gauges for detection. For our resonating membrane mass-flow sensor [19] as well as for our resonating microbridge mass-flow sensor [20], we make use of in situ phosphorus doping of low-pressure chemical vapour deposited (LPCVD) polysilicon for the excitation and the detection resistors, together with a single-crystal silicon substrate. We will first discuss the advantages of polysilicon for this application, and then we will give reasons for our choice of in situ doping with phosphorus.

Thin-film resistive elements, rather than diffusion wells, provide a freedom of choice of the material for the micromechanical structure. The thermal expansion coefficient of polysilicon is close to that of the single-crystal substrate, which minimizes thermal stresses building up with ambient temperature fluctuations. Moreover, semiconductors exhibit the piezoresistive effect, which provides strain gauges with a higher sensitivity than conductive materials. By doping the polysilicon and by growing the appropriate thickness, a desired sheet resistivity can be obtained. A small temperature coefficient of resistivity $(T C R)$ is desired in the application of resistors in resonating structures, as in that case the heat generation by dissipation is more convenient to control. It may be possible to obtain a $T C R$ close to zero by controlling the dopant concentration [5] or by applying the proper annealing treatment.

Doping can be achieved by drive-in diffusion, by ion implantation, or by in situ doping during the deposition process $[1,2]$. Within the field of micromechanics alone, aH three techniques are used for polysilicon films; the most suitable technique depends on the specific requirements, the dopant type, the overall fabrication process, and 


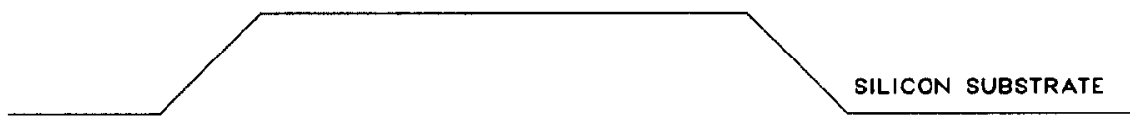

Fig. 1. Micromechanical resonator with elements for excitation and detection of the vibration.

on the available facilities. In the case of a resonating micromechanical device with resistors for thermal excitation and piezoresistive detection, the best option for practical applications is in situ doping. In contrast to ion implantation and drivein diffusion, in situ doping does not require additional processing other than annealing, leading to a minimum number of processing steps. Also, in situ doping readily yields a dopant profile which is flat across the thickness of the film, as well as a lower resistivity than can be obtained with postdeposition doping techniques.

Boron-doped polysilicon is known to have a higher strain sensitivity than phosphorus-doped polysilicon. However, as diborane is rapidly depleted in the low-pressure chemical vapour deposition reactor due to gas-phase reactions at elevated temperatures, we prefer to use phosphine for in situ doping.

\section{Materials Aspects}

\section{Deposition Process}

In the low-pressure chemical vapour deposition of polysilicon from a silane/ammonia gas mixture, the growth rate drops sharply if phosphine is introduced into the reactor as the source gas for in situ doping, and shows a large variation across each wafer [21]; the phosphine adsorbs much more competitively than the silane, with sticking coefficients of $s=1$ and $s=0.025$, respectively [22]. An explanation for this difference in adsorption is the presence of a lone pair of valence electrons in the phosphine molecule, and the full coordination of the silane molecule, enabling the phosphine to compete more successfully for available surface sites [21]. In this case the surface is fully covered by a passivating phosphine layer. The deposition rate is found to be strongly dependent on reactor geometry, wafer spacing, position of the wafer, and on the radial position on the wafer. The resulting thickness profile is characteristic of a transport-limited process [22], with depletion of active species in the inter-wafer region, superposed on a small uniform background deposition due to a contribution by an inter-wafer gas-phase reaction. Gas-phase silylene is assumed to be the reactive species; the silylene radical is able to compete successfully with the phosphine to reach the surface [23].

A $100 \%$ contribution by gas-phase reactions in the inter-wafer region would still result in a uniform growth rate. This is approximated by caging the wafers in slitted cassettes [24] or by a wafer spacing equal to the small gap between the wafer and the furnace tube [25]. During deposition, crystal nucleation and growth in the phosphorusdoped film occur at the gas-film interface [26], leading to a transition temperature between amorphous and crystalline films as low as $520^{\circ} \mathrm{C}$. The in situ phosphorus-doped polysilicon is annealed in a nitrogen environment for diffusion of phosphorus atoms to lattice sites and for recrystallization $[2,24,27-30]$, where phosphorus atoms enhance grain growth $[2,29]$.

As a theoretical model for the conductivity of polysilicon, a-carrier trapping model was first qualitatively proposed by Kamins [31], and was quantitatively evaluated by Seto [32]. In this model the grain boundary, which is only a few atomic layers thick, contains trapping states caused by defects resulting from disordered or incomplete atomic bonding. These states trap part of the free charge carriers from the ionized and uniformly distributed dopants. This process creates a depletion layer, which is now also a spacecharge region, along the grain boundary.

The effective resistance of the polycrystalline material consists of contributions from the grainboundary regions and from the undepleted cores of the grains. At high dopant concentrations positive temperature coefficient(PTC)-like behaviour [33] is observed in experiments [31,32]. Although Kamins [31] supposes this to be caused by scattering as is found in single-crystal silicon, Seto [32] shows that the resistivity of the depletion region also shows this PTC-behaviour, according to the known temperature dependence of the thermionic emission current, combined with a low barrier height for narrow depletion regions, i.e., for high dopant concentrations.

\section{Gauge Factor}

The change in resistance of a strain gauge due to strain $\varepsilon$ (in general a first-order tensor with six components) is caused by a change in the dimensions of the resistor and, for piezoresistive materi- 
als, by a change in resistivity due to the accompanying mechanical stress $\sigma(\varepsilon)[33,34]$. For singlecrystalline silicon, the change in conductivity due to mechanical stress is caused by a shift in the relevant conduction and valence band minima and maxima relative to each other, resulting in a redistribution of charge carriers between minima or bands [33]. This piezoresistance effect is anisotropic and reduces with increasing carrier concentration [33, 34].

The gauge factor of a material is then defined as the relative change in resistance per unit strain, usually in a stress situation of uniaxial stress, see Fig. 2(a). However, if the strain gauge and the substrate have different Poisson's ratios, the elastic properties of the substrate form a parameter in determining the gauge factor. Therefore, it is more practical to define the gauge factor for a situation of planar stress with in-plane uniaxial strain, see Fig. 2(b), as is the case in pure bending of a plate to a cylindrical surface [35]. This definition is also applied in ref. 36.

For the modelling of the piezoresistive effect in polysilicon, the above trapping model with Schottky-type barriers is commonly used. In most models $[8,36-38]$ the effect of mechanical stress on the grain boundary behaviour is neglected, and the change in resistivity is dominated by the anisotropic piezoresistive effect of single-crystalline silicon in the undepleted core. In ref. 39 this model is extended by including the effect of mechanical stress on the mechanism of thermionic emission.

The quantitative models make use of averaging the contributions of separate grains with different
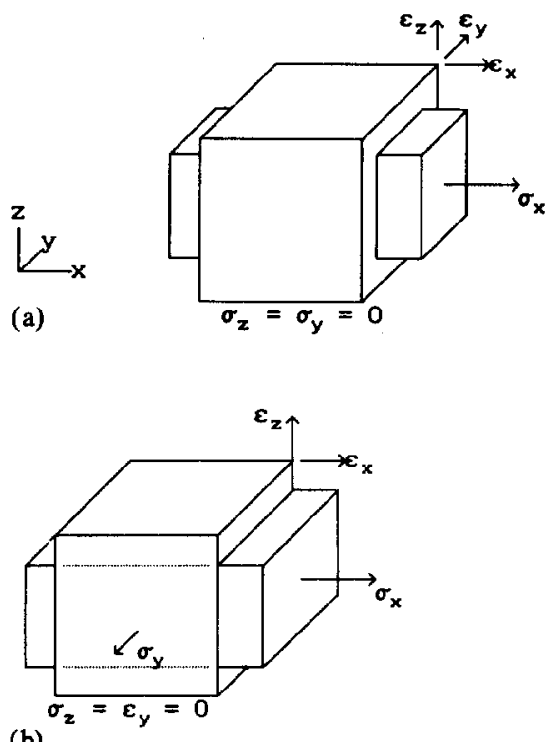

(b)

Fig. 2. (a) Uniaxial stress and (b) planar stress with in-plane uniaxial strain. orientations, resulting in theoretical gauge factors for films with randomly oriented crystallites, as well as for textured films. The observed behaviour is explained reasonably well by the first model $[8,36-38]$, whereas the behaviour of films with smaller dopant concentrations and/or with smaller grain size is explained better by the extended model [39]. For a textured film dominated by $\langle 110\rangle,\langle 331\rangle,\langle 111\rangle$ and $\langle 311\rangle$ orientations, and with a grain size of $190 \mathrm{~nm}$, ref. 39 predicts maximum values for the theoretical gauge factor for uniaxial stress of -22 for a phosphorusdoped film at a dopant concentration $N_{\mathrm{D}}=5 \times 10^{19} \mathrm{~cm}^{-3}$, and of +29 for a borondoped film at $N_{\mathrm{D}}=1.6 \times 10^{19} \mathrm{~cm}^{-3}$. For larger grain sizes higher gauge factors are predicted.

\section{Experiments}

\section{Sample Preparation}

2 " (100) n-type silicon wafers were used as substrates. These were oxidized for $15 \mathrm{~min}$ in a wet environment at $1150^{\circ} \mathrm{C}$ to an oxide thickness of $300 \mathrm{~nm}$. Low-pressure chemical vapour deposition of in situ phosphorus-doped polysilicon was performed in a conventional $4^{\prime \prime}$ quartz furnace tube, with $3^{\prime \prime}$ dummy wafers back to back $2 \mathrm{~mm}$ behind each 2 " wafer, to create a narrow slit effect for the gas flow, see Fig. 3.

Process parameters were taken from a recipe optimized for low resistivity and a uniform growth rate [40]: pressure 1 mbar, silane mass flow $60 \mathrm{sccm}$, phosphine (2000 ppm) mass flow $30 \mathrm{sccm}$, nitrogen mass flow $300 \mathrm{sccm}$, deposition time $80 \mathrm{~min}$, and deposition temperature $625^{\circ} \mathrm{C}$ at the centre of the heated zone, with a temperature ramp of $10^{\circ} \mathrm{C}$ across the heated zone for the compensation of gas-phase depletion. Three series of samples were grown. Series A was grown using the above recipe, yielding an average film thickness of $370 \mathrm{~nm}$. Series B was grown with the same process parameters as series $A$, but with a pressure of 0.275 mbar, yielding an average film thickness of $170 \mathrm{~nm}$. Series $C$ was grown with the standard process parameters again, but in a $3^{\prime \prime}$

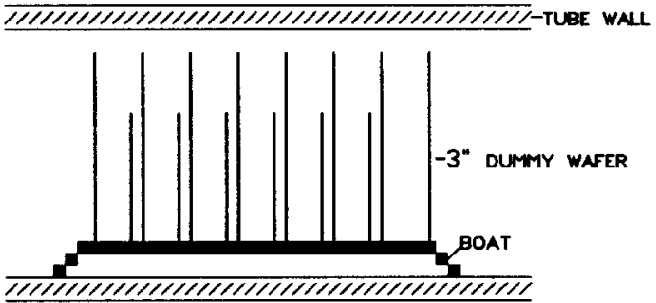

Fig. 3. Narrow slit effect for $2^{\prime \prime}$ wafers in a $4^{\prime \prime}$ tube, using $3^{\prime \prime}$ dummy wafers. 
tube and without the $3^{\prime \prime}$ dummy wafers, yielding an average film thickness of $400 \mathrm{~nm}$.

The samples were annealed in a furnace tube in a nitrogen environment at different temperatures ranging from $800^{\circ} \mathrm{C}$ to $1150^{\circ} \mathrm{C}$ for $30 \mathrm{~min}$, i.e., without ramping of the furnace temperature.

After annealing of the polysilicon, an aluminium (series $\mathbf{A}$ and $\mathbf{B}$ ) or a chromium-gold metal film (series C) was grown by evaporation. The metal film and the polysilicon were patterned to form resistors with connection pads. Finally, the wafer was diced into separate bar-shaped samples. The length of the polysilicon resistor in-between the two metal pads was $1 \mathrm{~mm}$, with a width of either 100 or $200 \mu \mathrm{m}$.

\section{Measurements}

Thickness measurements of the polysilicon films were carried out by measuring the step heights in the polysilicon after patterning, using a surface profiler (Dektak 3030). Thickness profiles were determined for each wafer. From these the thickness $t$ at the centre and the standard deviation $\sigma$ were determined, as well as the average increase $\Delta$ in $t$ from wafer to wafer, see Table 1 . A standard deviation from a uniform thickness of 1 to $2 \%$ was found for series $A$ and $B$, and of $10 \%$ for series C. Measurement scans from wafer to wafer showed an increasing thickness of the polysilicon thin film, linear with the position downstream in the reactor tube, increasing by $4 \%$ (series $\mathrm{A}$ and $\mathrm{C}$ ) to $8 \%$ (series $\mathrm{B}$ ) from wafer to wafer. The surface roughness $\delta$ (average peak-tovalley heights) was found to be $12 \mathrm{~nm}$ for series $A$ and $C$ and $10 \mathrm{~nm}$ for series $B$.

To determine the grain size, samples of series $A$ and of series $C$ were prepared for SEM analysis.
The samples were broken at room temperature, and the flaw was treated in a 1:5 Wright etch:water solution for $\mathbf{4 0} \mathrm{s}$ to improve the contrast of the grain boundaries [41]. Average grain sizes of 20 to $30 \mathrm{~nm}$ were observed for both series, with no difference between annealed and unannealed samples. Figure 4 shows an SEM photograph of a sample of series $A$, annealed at $850^{\circ} \mathrm{C}$ for $30 \mathrm{~min}$. The phosphorus concentration was determined by SIMS analysis, using a caesium source. Samples of series $\mathrm{A}$ and $\mathrm{C}$ exhibited a dopant concentration of $2 \times 10^{20} \mathrm{~cm}^{-3}$ and of $3 \times 10^{20} \mathrm{~cm}^{-3}$, respectively, both independent of

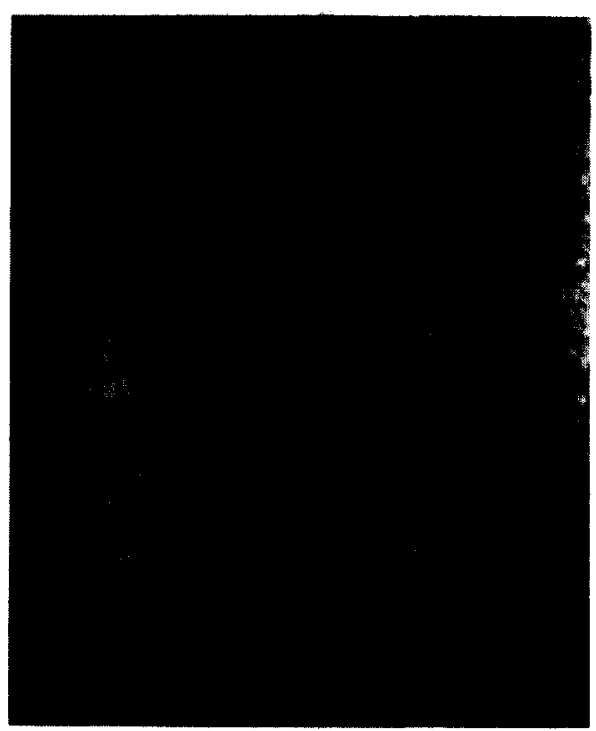

Fig. 4. SEM photograph of in situ phosphorus-doped polysilicon film on oxidized silicon substrate (series $A$, annealed at $850^{\circ} \mathrm{C}$ for $30 \mathrm{~min}$ ).

TABLE 1. Summary of experimental results for samples from all three series; Parameter $x$ indicates the position of the wafer during deposition, with the number increasing downstream. Thickness $t$ is taken at the centre of the wafer, $\sigma$ is the standard deviation from a uniform thickness, while $\Delta$ is the average increase in thickness with downstream position, and $\delta$ is the surface roughness (average peak-to-valley height). Annealing was done for $30 \mathrm{~min} ; \rho, T C R, k$ and $T C k$ are averaged across the wafer

\begin{tabular}{|c|c|c|c|c|c|c|c|c|c|}
\hline Series & $L, N_{\mathrm{D}}$ & $x$ & $t(\mathrm{~nm})$ & $\sigma, \Delta, \delta$ & $\begin{array}{l}T_{\text {anneal }} \\
\left({ }^{\circ} \mathrm{C}\right)\end{array}$ & $\begin{array}{l}\rho \\
(\mathrm{m} \Omega \mathrm{cm})\end{array}$ & $\begin{array}{l}\operatorname{TCR} \\
\left(10^{-4} /{ }^{\circ} \mathrm{C}\right)\end{array}$ & $-k$ & $\begin{array}{c}-T C k \\
\left(\% /{ }^{\circ} \mathrm{C}\right)\end{array}$ \\
\hline $\begin{array}{l}\text { A } \\
4^{\prime \prime} \text { tube } \\
1 \text { mbar }\end{array}$ & $\begin{array}{l}20-30 \mathrm{~nm} \\
2 \times 10^{20} \mathrm{~cm}^{-3}\end{array}$ & $\begin{array}{l}8 \\
5 \\
7\end{array}$ & $\begin{array}{l}400 \\
330 \\
360\end{array}$ & $\begin{array}{c}1-2 \% \\
4 \% \\
12 \mathrm{~nm}\end{array}$ & $\begin{array}{l}800 \\
875 \\
950\end{array}$ & $\begin{array}{l}7.9 \\
2.0 \\
1.1\end{array}$ & $\begin{array}{l}-7.5 \\
-5.0 \\
+9.2\end{array}$ & $\begin{array}{l}27.5 \\
20.3 \\
18.8\end{array}$ & 0.25 \\
\hline $\begin{array}{l}\text { B } \\
4^{\prime \prime} \text { tube } \\
0.275 \\
\text { mbar }\end{array}$ & & $\begin{array}{l}6 \\
1 \\
4 \\
2 \\
3 \\
5\end{array}$ & $\begin{array}{l}170 \\
140 \\
140 \\
140 \\
150 \\
170\end{array}$ & $\begin{array}{c}1-2 \% \\
4 \% \\
10 \mathrm{~nm}\end{array}$ & $\begin{array}{r}800 \\
850 \\
900 \\
1000 \\
1150\end{array}$ & $\begin{array}{r}26.0 \\
15.0 \\
8.7 \\
2.8 \\
1.5 \\
1.3\end{array}$ & $\begin{array}{r}-18.2 \\
-10.1 \\
-6.3 \\
+3.6 \\
+6.0 \\
+6.2\end{array}$ & $\begin{array}{l}28.4 \\
27.8 \\
23.4 \\
22.0 \\
20.1 \\
19.7\end{array}$ & $\begin{array}{l}0.3 \\
0.25 \\
0.2\end{array}$ \\
\hline $\begin{array}{l}\text { C } \\
\text { 3" tube } \\
1 \text { mbar }\end{array}$ & $\begin{array}{l}20-30 \mathrm{~nm} \\
3 \times 10^{20} \mathrm{~cm}^{-3}\end{array}$ & $\begin{array}{l}1 \\
2\end{array}$ & $\begin{array}{l}390 \\
410\end{array}$ & $\begin{array}{c}10 \% \\
8 \% \\
12 \mathrm{~nm}\end{array}$ & $\begin{array}{l}800 \\
900\end{array}$ & $\begin{array}{l}9.0 \\
3.8\end{array}$ & $\begin{array}{l}-6.5 \\
+1.5\end{array}$ & 15.0 & \\
\hline
\end{tabular}


the annealing treatment. These values are close to the solid solubility of phosphorus in silicon (3.5 $\times$ $10^{20} \mathrm{~cm}^{-3}$ [2]). Unfortunately, we did not conduct the above experiments on samples of series $B$.

The sheet resistances of the polysilicon films were measured using a conventional four-point probe. From these, together with the above thickness measurements, the resistivity was determined. Figure 5(a) shows the resistivity of the polysilicon film of series B (averaged from five measurements near the centre of the wafer) as a function of anneal time after annealing at $850^{\circ} \mathrm{C}$ for successive time intervals. Note that the effective anneal time is difficult to quantify due to heating and cooling effects when the sample is moved into and out of the furnace. Two separate regimes can be recognized. The curve shows a sharp decrease of the resistivity within the first $30 \mathrm{~s}$ of annealing, and a diminishing decrease with longer anneal times. Figure 5(b) shows the resistivity of films of series $B$ as a function of anneal temperature, after annealing for $30 \mathrm{~min}$. For anneal temperatures between 800 and $900^{\circ} \mathrm{C}$ it shows a strong decrease of resistivity with increasing anneal temperature, with this decrease diminishing for higher anneal tempeatures.

The resistance of the polysilicon resistors was measured using a four-point contact arrangement as shown in Fig. 6. A current $I$ was supplied to

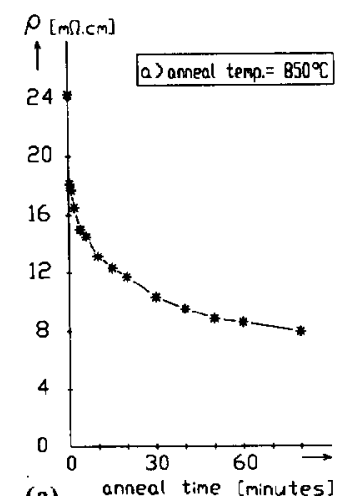

(a) anneal time [minutes]

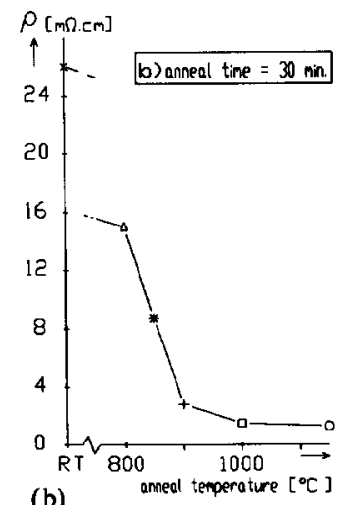

(b)
Fig. 5. Resistivity of films of series $B$ (a) as a function of anneal time after annealing at $850^{\circ} \mathrm{C}$ and (b) as a function of anneal temperature after annealing for $\mathbf{3 0} \mathrm{min}$.

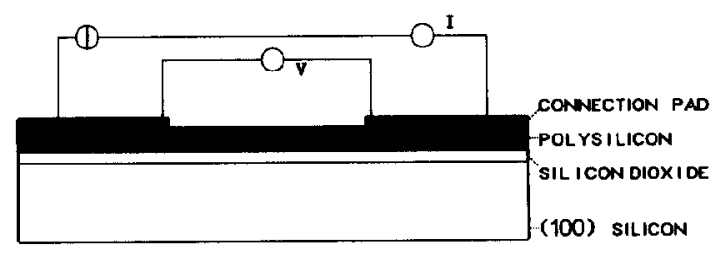

Fig. 6. Four-point contact arrangement for resistance measurements. the outer two contacts. The two centre contacts for voltage measurement $V$ were located close to the edges of the metal pads. This way, the effect of contact resistances between the metal film and the polysilicon, as well as other series resistances, was minimized. The total resistance between the two centre contacts is then found from $R=V / I$. (Note that for a proper four-point measurement the two metal pads need to be divided into four separate electrodes. The centre two electrodes for the voltage measurement then need to be of a width of the order of the thickness of the polysilicon film. This would require submicron technology.) Additional resistance measurements were carried out with polysilicon resistors with lengths of $100 \mu \mathrm{m}$ and $200 \mu \mathrm{m}$ (series A, annealed at $875^{\circ} \mathrm{C}$ for $30 \mathrm{~min}$ ). These revealed by extrapolation a total series resistance of less than $10 \Omega$, which is negligible. $I-V$ and impedance measurements (with a lower limit of $10 \mu \mathrm{A}$ ) indicated no diode characteristics, and no parasitic capacitance or induction from d.c. up to a $100 \mathrm{kHz}$ harmonic current. The resistivity of the polysilicon was then determined from the measured resistance and the known dimensions. For the latter the thickness was determined as described above, and the width and the length of the resistors were measured using a microscope.

To measure the temperature dependence of the resistivity, the samples, together with a reference sample with a thermocouple, were mounted on a copper plate. With the aid of a Peltier element, the temperature was varied from $-10^{\circ} \mathrm{C}$ to $100^{\circ} \mathrm{C}$. For temperatures below $-10^{\circ} \mathrm{C}$ the sample was cooled by sprinkling it with liquid nitrogen. Figure 7 (a) shows the experimental results, with the values for the resistances normalized to the value at room temperature $\left(20^{\circ} \mathrm{C}\right)$. The measured data were fitted with a polynomial using least squares.

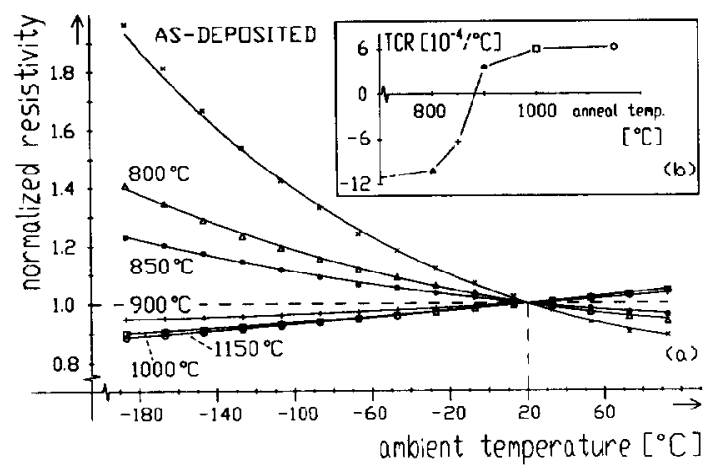

Fig. 7. (a) Resistivity of films of series $B$ as a function of ambient temperature, after annealing for $30 \mathrm{~min}$ at different anneal temperatures, normalized for the resistivity at room temperature; (b) temperature coefficient of resistivity (TCR) as a function of anneal temperature. 


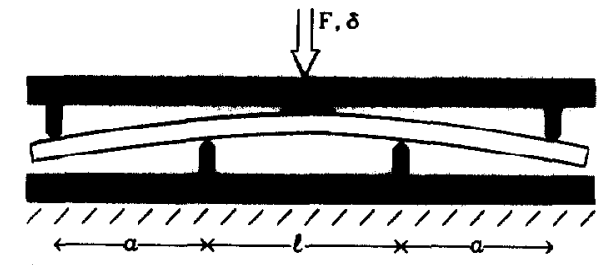

(a)

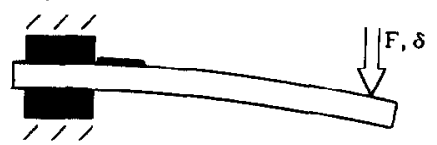

(b)

Fig. 8. Schematic representation of (a) the four-point bending test assembly and (b) a one-point cantilever test assembly.

The $T C R$ at room temperature was then determined from the slope of the fitted curve. These values are represented in Fig. $7(\mathrm{~b})$ as a function of anneal temperature.

Gauge factor measurements were carried out using a four-point bending test, see Fig. 8(a); this consists of two stamps, both with a pair of knife edges. The two stamps sandwich the sample, using a plastic foil to insulate the connection pads from the knife edges. The lower stamp is rigidly supported, and the upper stamp is loaded by a transverse force $F$ at its centre. The downward displacement $\delta$ of the upper stamp is controlled using a micro-control. If the stamps are properly aligned, a pure bending moment $M=F a$ is exerted on the part of the sample in-between the central knife edges, where $a$ is half the difference of the separations between the knife edges in the upper stamp and the lower stamp.

As the width of the sample is much larger than its thickness, the sample bends like a plate, i.e., a planar stress with (in-plane) uniaxial strain rather than a uniaxial stress, see Fig. 2 [35]. As length $a$ is much larger than the thickness of the sample, deflection due to mechanisms other than bending is negligible. For the downward displacement $\delta$ of the upper stamp, the longitudinal normal strain $\varepsilon_{x}$ of the thin-film resistor is then found from

$\varepsilon_{x}=\frac{\delta t_{\mathrm{s}}}{l a} /\left[1+\frac{2 a}{3 l}\right]$

where $l$ is the separation between the central knife edges and $t_{\mathrm{s}}$ is the thickness of the substrate underneath the resistor, i.e., not including the resistor itself. In deriving eqn. (1) it was assumed that the sample can be regarded as a prismatic beam.

Unlike the one-point cantilever bending test, see Fig. $8(\mathrm{~b})$, this four-point bending test has a low sensitivity for misalignments. Misalignments of the stamps and of the micro-control only lead to second-order effects in the occurring strain, whereas effects in the one-point cantilever bending test lead to first-order effects. Moreover, once the micro-control and the two stamps of the fourpoint bending test assembly have been aligned properly, a misalignment of the sample has no effect in this arrangement, as long as the strain gauge is in-between the central knife edges.

The strain was scanned from $-\mathbf{0 . 8 \%}$ to $+0.8 \%$, where no distinguishable hysteresis occurred. The temperature coefficient of the gauge factor, $T C k \equiv \Delta|k| /|k| \Delta T$, was determined by simply increasing and lowering the ambient temperature five degrees above and below room temperature. Figure 9 shows results of measurements on the gauge factor of samples of series B. Table 1 summarizes the experimental results for samples of all three series.

\section{Discussion}

Wafers from series $C$ showed a thickness profile characteristic for a process with a contribution from transport kinetics, although the small gap between the edge of each wafer and the reactor wall limited this contribution to $10 \%$ of the uniform contribution. The arrangement of Fig. 3 for series A and B improved the uniformity of the growth rate across individual wafers to within 1 to $2 \%$ standard deviation. The thickness increases with downstream position by $4 \%$ for series $A$ and $B$, and by $8 \%$ for series C. Apparently, the downstream depletion of the gas phase was over-compensated by the applied temperature ramp. The surface roughness (average peak-tovalley heights) amounts to $12 \mathrm{~nm}$ for series $A$ and $C$, and $10 \mathrm{~nm}$ for series $B$.

The grain size and dopant concentration were measured for samples from series $A$ and $C$. No distinguishable difference in grain size was de-

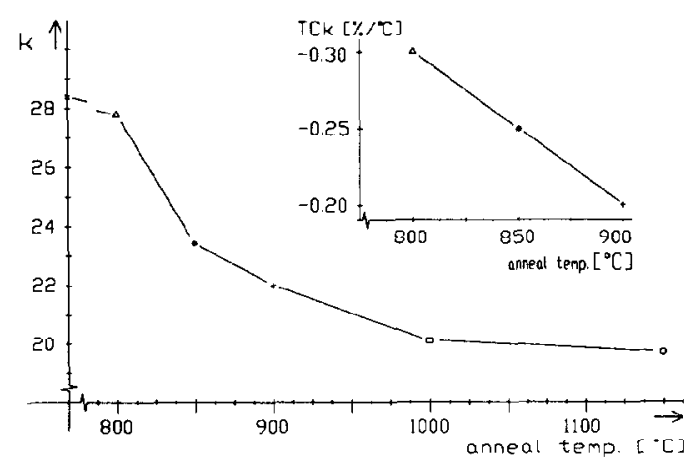

Fig. 9. Experimentally determined gauge factor $k$, and its temperature coefficient $T C k$ (insert) of films of series B, as a function of anneal temperature. 
tected before and after annealing, with a grain size of 20 to $30 \mathrm{~nm}$ for both series. During the deposition process, the nucleation rate apparently was high compared to the (surface) diffusion velocity, leading to small grains in the as-deposited film. The absence of recrystallization during annealing may be due to the large number of grains, and to the homogeneous distribution of their sizes. The dopant concentration was slightly higher for series $C$ than for series $A$, $3 \times 10^{20} \mathrm{~cm}^{-3}$ and $2 \times 10^{20} \mathrm{~cm}^{-3}$, respectively. For a trapping density $Q_{\mathrm{t}}$ of $4 \times 10^{12} \mathrm{~cm}^{-2}$, this implies a partial depletion with a width $w$ of the depletion layer of the order of only $0.2 \mathrm{~nm}$ (theoretically), which is still much smaller than the grain size. Hence, the electrical properties of the polycrystalline film will be dominated by those of the single-crystal cores.

The measured values for the resistivity and for the temperature coefficient are of the same order of magnitude as those found in the literature $[23,24,29,30,32]$. The strong decrease in resistivity after annealing for $30 \mathrm{~s}$ or less, or after annealing at low temperatures, see Fig. 5(a) and (b), is caused by the movement of phosphorus atoms to lattice sites. The strong decrease of the (absolute) value of the $T C R$ is also qualitatively explained by this effect. The fact that the decrease of the resistivity was less pronounced after longer anneal times, or after annealing at increased temperatures, is indicative of a second mechanism. According to refs. 2, 24 and 28, this is related to grain growth. The dependence of the $T C R$ on annealing parameters can also be understood in this way. However, according to our SEM analysis, grain growth does not occur in our films. Alternatively, the data in Fig. 5(a) typically agree with a diffusion model. The observed dependence of resistivity on anneal time indicates a diffusion time of the order of $10^{3} \mathrm{~s}$. For a diffusion length of $10 \mathrm{~nm}$, this indicates a diffusion coefficient of the order of $10^{-15} \mathrm{~cm}^{2} / \mathrm{s}$, which is three orders magnitude lower than the diffusion coefficient of phosphorus atoms in silicon [2]. We will come back to this point at the end of this Section.

The gauge factor for the phosphorus-doped polysilicon film was negative, as expected, the absolute value decreasing with increasing anneal temperature, and stabilizing at higher temperatures: just below 19 for series $A$ and just below 20 for series $B$. For series $C$, the gauge factor was measured for only one sample, and was found to have an absolute value as low as $\mathbf{1 5}$. The observed difference of this series with series $A$ and $B$ could be due to a different texture. The temperature coefficient of the gauge factor was measured to be approximately $-0.25 \% /{ }^{\circ} \mathrm{C}$, slightly decreasing with decreasing gauge factor. The negative sign indicates that the absolute value of the gauge factor decreases with increasing temperature. These values for the gauge factor and its temperature coefficient are similar to those found by other authors [39].

According to the models presented in the literature, grain growth during annealing would have resulted in an increase of the gauge factor with increasing anneal temperatures. Viewed in this light, the fact that for our films the gauge factor decreased with increasing anneal temperature is in accordance with the non-appearance of grain growth. Instead, the diffusion of dislocations during annealing could explain this observed decrease of the gauge factor: less lattice defects within the crystallite mean that less charge carriers will be trapped, i.e., the concentration of free charge carriers will be higher. The latter causes a decrease of the gauge factor [33]. The decrease of the resistivity itself with increasing anneal temperature, as well as the observed dependence of its temperature coefficients, are also qualitatively explained by this model of diffusion of dislocations.

\section{Conclusions}

Three series of samples of LPCVD in situ phosphorus-doped polysilicon films have been grown under different process conditions. Polycrystalline films with grain sizes of 20 to $30 \mathrm{~nm}$, both before and after annealing, were obtained, with dopant concentrations of (23) $\times 10^{20} \mathrm{~cm}^{-3}$. A uniform thickness profile across each wafer was obtained by restricting the supply of active species to the inter-wafer regions. Films with low resistivities of the order of $1 \mathrm{~m} \Omega \mathrm{cm}$ were obtained after annealing at moderate temperatures for $30 \mathrm{~min}$. NTC-behaviour changed to PTC-behaviour after annealing at temperatures above $900{ }^{\circ} \mathrm{C}$. Negative values for the longitudinal gauge factor were measured, and were found to depend on anneal temperature, stabilizing at a value of approximately -20 . The strain-sensitive effect decreased with increasing ambient temperature by approximately $0.25 \%$ / ${ }^{\circ} \mathrm{C}$. The decrease of both the resistivity and the gauge factor with increasing anneal temperature, in the absence of grain growth, suggests a mechanism in which free charge carriers are generated during annealing. One of the underlying mechanisms may be related to the diffusion of dislocations. Although the grain size is very small, the electrical properties of the polycrystalline film are dominated by the bulk of the crystallites, according to the trapping state model. 
With the above values for the resistivity and for the gauge factor, and the possibility of obtaining a $T C R$ close to zero, these films are appropriate for use in thermal excitation and piezoresistive detection in micromechanical resonators.

\section{Acknowledgements}

The authors would like to thank Rob Legtenberg for preparing the samples, and $\mathrm{Mr}$ Vandervos of IMEC, Leuven, Belgium, for carrying out the SIMS analysis. We also would like to thank the staff of the IC-laboratory of the Department of Electrical Engineering of the University of Twente for letting us use their low-pressure chemical vapour deposition system, and especially Jisk Holleman for his many helpful suggestions. Also, we would like to thank Professor Theo Popma for many thoughtful discussions. Finally, we would like to thank Ms Katrina Emmett for carefully reading the manuscript.

\section{References}

1 A. C. Adams, Dielectric and polysilicon deposition, in S. M. Sze (ed.), VLSI Technology, McGraw-Hill, New York, 1983, Ch. 3, pp. 93-130.

2 T. I. Kamins, Polycrystalline Silicon for Integrated Circuit Applications, Kluwer, Dordrecht, 1988, pp. 1, 31-35, 91106.

3 E. Lüder, Polycrystalline silicon-based sensors, Sensors and Actuators, 10 (1986) 9-23.

$4 \mathrm{~J}$. M. Jaffe, Monolithic polycrystalline silicon pressure transducer, Electron. Lett., 10 (1974) 420-421.

5 E. Obermeier, P. Kopystinski and R. Niessl, Characteristics of polysilicon layers and their application in sensors, IEEE Solid-State Sensor Workshop, Hilton Head, SC, U.S.A., June 6-9, 1986. See also E. Obermeier, Polysilicon layers lead to a new generation of pressure sensors, Proc. 3 rd Int. Conf. Solid-State Sensors and Actuators (Transducers '85), Philadelphia, PA, U.S.A., June 11-14, 1985, pp. 527-536.

6 W. Benecke, L. Csepregi, A. Heuberger, K. Kühl and H. Seidel, A frequency-selective, piezoresistive silicon vibration sensor, Proc. 3rd Int. Conf. Solid-State Sensors and Actuators (Transducers '85), Philadelphia, PA, U.S.A., June II14, 1985, pp. 105108.

7 H. Guckel, D. W. Burns, C. R. Rutigliano, D. K. Showers and J. Uglow, Fine grained polysilicon and its application to planar pressure transducers, Proc. 4th Int. Conf. SolidState Sensors and Actuators (Transducers '87), Tokyo, Japan, June 2-5, 1987, pp. 277-280.

8 J. Suski, V. Mosser and J. Goss, Polysilicon SOI pressure sensor, Sensors and Actuators, 17 (1989) 405-414.

9 H. Schäfer, V. Graeger and R. Kobs, Temperature-independent pressure sensors using polycrystralline silicon strain gauges, Sensors and Actuators, 17 (1989) 521-527.

10 Y.C. Tai and R. S. Muller, Lightly-doped polysilicon bridge as a flow meter, Sensors and Actuators, 15 (1988) 63-75.

11 R. T. Howe and R. S. Muller, Resonant microbridge vapor sensor, IEEE Trans. Electron Devices, ED-33 (1986) 499506.

12 S. F. Bart, T. A. Lober, R. T. Howe, J. H. Lang and M. F. Schlecht, Design considerations of micromachined electric actuators, Sensors and Actuators, 14 (1988) 269-292.
13 M. Mehregany, K. J. Gabriel and W. S. N. Trimmer, Integrated fabrication of polysilicon mechanisms, IEEE Trans. Electron Devices, 35 (1988) 719-723.

14 L. S. Fan, Y. C. Tai and R. S. Muller, Integrated movable micromechanical structures for sensors and actuators, IEEE Trans. Electron Devices, 35 (1988) 724-730.

15 W. C. Tang, T.-C. H. Nguyen and R. T. Howe, Laterally driven polysilicon resonant microstructures. Sensors and Actuators, 20 (1989) 25-32. See aiso Proc. IEEE Micro Electro Mechanical Systems Workshop (MEMS-89), Salt Lake City, UT, U.S.A., Feb. 1989, pp. 53-59.

16 C. Linder and N. F. de Rooij, Investigations on free-standing polysilicon beams in view of their application as resonators, Sensors and Actuators, A21-A23 (1990) 1053-1059. Se also Tech. Digest, Sth Conf. Solid-State Sensors and Actuators (Transducers '89), Montreux, Switzerland, June 25-30, 1989, pp. 289-290.

17 H. Guckel, D. W. Burns, C. C. G. Visser, H. A. C. Tilmans and D. Deroo, Fine-grained polysilicon films with built-in tensile strain, IEEE Trans. Electron Devices, 35 (1988) $800-801$.

18 R. S. Hijab and R. S. Muller, Residual strain effects on large aspect ratio micro-diaphragms, Proc. IEEE Micro Electro Mechanical Systems Workshop (MEMS-89), Salt Lake City, UT, U.S.A., Feb. 1989, pp. 133-138.

19 S. Bouwstra, P. Kemna and R. Legtenberg, Thermally excited resonating membrane mass flow sensor, Sensors and Actuators, 20 (1989) 213-223.

20 S. Bouwstra, R. Legtenberg, H. A. C. Tilmans and $M$. Elwenspoek, Resonating microbridge mass flow sensor, Sensors and Actuators, A2I-A23 (1990) 332-335.

21 B. S. Meyerson and W. Olbricht, Phosphorus-doped polycrystalline silicon via LPCVD, I. Process Characterization, J. Electrochem. Soc., 131 (1984) 2361-2365.

22 B. S. Meyerson and M. L. Yu, Phosphorus-doped polycrystalline silicon via LPCVD, II. Surface interactions of the silane/phosphine/silicon system, J. Electrochem. Soc., 131 (1984) 2366-2368.

23 A. J. Learn and D. W. Foster, Deposition and electrical properties of in situ phosphorus-doped silicon films formed by LPCVD, J. Appl. Phys., 6I (1987) 1898-1904.

$24 \mathrm{~J}$. Mulder, LPCVD of phosphorus-doped polysilicon, Proc. 3rd Symp. Sensors and Actuators, Enschede, The Netherlands, Oct. 1986, pp. 75-84.

$25 \mathrm{~A}$. Yeckel and $\mathrm{S}$. Middleman, A model of growth rate nonuniformity in the simultaneous deposition and doping of a polycrystalline silicon film by LPCVD, J. Electrochem. Soc., 134 (1987) 1275-1281.

26 D. Bielle-Daspet, L. Mercadere and M. Boukezzata, Structural and technological properties of heavily in situ phosphorus-doped LPCVD silicon films, Thin Solid Films, 168 (1989) 43-48

27 T. I. Kamins, M. M. Mandurah and K. C. Saraswat, Structure and stability of LPCVD silicon films, J. Electrochem. Soc., 125 (1978) 927-932.

28 M. M. Mandurah, K. C. Saraswat and T. I. Kamins, Phosphorus doping of LPCVD silicon films, J. Electrochem. Soc., 126 (1979) 1019-1023.

29 G. Harbeke, L. Krausbauer, E. F. Steigmeier, A. E. Widmer, H. F. Kappert and G. Neugebauer, LPCVD polycrystalline silicon: growth and physical properties of in situ phosphorus doped and undoped films, $R C A$ Rev., 44 (1983) 287-312.

30 M. M. Mandurah, K. C. Saraswat, C. R. Helms and T. I. Kamins, Dopant segregation in polycrystalline silicon, $J$. Appl. Phys., 51 (1980) 5755-5763.

31 T. I. Kamins, Hall mobility in chemical deposited polycrystalline silicon, J. Appl. Phys., 42 (1971) 4357-4365.

$32 \mathrm{~J}$. Y. W. Seto, The electrical properties of polycrystalline silicon films, J. Appl. Phys., 46 (1975) 5247-5254. 
33 W. P. Mason, Semiconductor transducers - general considerations, in W. P. Mason (ed.), Physical Acoustics, Academic Press, New York, 1964, Ch. 10, p. 173.

34 Y. Kanda, A graphical representation of the piezoresistive coefficients in silicon, IEEE Trans. Electron Devices, ED-29 (1982) 64-70.

35 S. P. Timoshenko and S. Woinowski-Krieger, Theory of Plates and Shells, McGraw-Hill, New York, 1970, p. 1.

36 D. Schubert, W. Jenschke, T. Uhlig and F. M. Schmidt, Piezoresistive properties of polycrystalline and crystalline silicon films, Sensors and Actuators, 11 (1987) 145-155.

37 J. Y. W. Seto, Piezoresistive properties of polycrystralline silicon, J. Appl. Phys., 47 (1976) 4780-4783.

38 P. J. French and A. G. R. Evans, Piezoresistance in polysilicon, Electron. Lett., 20(24) (1984) 999-1000.

39 P. J. French and A. G. R. Evans, Polycrystalline silicon strain sensors, Sensors and Actuators, 8 (1985) 219-225; Polycrystalline silicon as a strain gauge material, $J$. Phys. E: Sci. Instrum., 19 (1986) 1055-1058.

$40 \mathrm{~J}$. Holleman, personal communication.

41 M. Wright-Jenkins, A new preferential etch for defects in silicon crystals, J. Electrochem. Soc., 124 (1977) 757-762.

\section{Biographies}

Siebe Bouwstra was born in Amsterdam, The Netherlands, in 1958. He received his M.Sc. degree in mechanical engineering from the University of Twente, Enschede, The Netherlands, in 1984, with major and minor subjects in applied mechanics and in tribology, respectively. In the same year he joined the Sensors and Actuators Research Unit at the University of Twente as a contract researcher, where his research focused on micromechanical resonating structures. In the first two years he developed a prototype laser beam scanner for an industrial contract. In 1986 he started a four-year project to develop a resonating microbridge mass flow sensor and in March 1990 he received his Ph.D. with a thesis based on this research. As a fellow of the Dutch Academy of Sciences he is engaged in starting up a research project on surface micromachined resonating structures for sensor and actuator applications.

Eddy de Weerd was born in Groningen, The Netherlands, in 1958. He received his M.Sc. degree in electrical engineering from the University of Twente, Enschede, The Netherlands, in 1989, with his master's thesis based on in situ phosphorus-doped polysilicon. He is presently working for Twente Technology Transfer, Enschede, The Netherlands, where he is engaged in the development of a micromechanical microphone.

Miko Elwenspoek was born in Eutin, F.R.G., in 1948. He received his M.Sc. degree in physics from the Free University of Berlin, F.R.G., in 1978. He received his doctor's degree from the Free University of Berlin in 1983, with his thesis based on liquid alloys. From 1983 to 1987 he worked at the Catholic University of Nijmegen, The Netherlands, where his research focused on crystal growth from a solution. In 1987 he joined the micromechanics group of the Sensors and Actuators Research Unit at the University of Twente, The Netherlands. 